\title{
Topographic mapping of the Mars MC quadrangles using HRSC data
}

\author{
E. Kersten ${ }^{\mathrm{a}, *}$, K. Gwinner ${ }^{\mathrm{a}}$, G. Michael ${ }^{\mathrm{b}}$, A. Dumke ${ }^{\mathrm{b}}$, J. Bostelmann ${ }^{\mathrm{c}}$ ***, R. Jaumann ${ }^{\mathrm{b}}$, and the \\ HRSC Global Topography and Mosaic Generation Task Group \\ ${ }^{a}$ Institute of Planetary Research, German Aerospace Center (DLR), Germany, contact: elke.kersten@dlr.de \\ ${ }^{b}$ Institute of Geological Sciences, Freie Universität Berlin, Germany \\ ${ }^{c}$ Institute of Photogrammetry and GeoInformation, Leibniz Universität Hannover, Germany \\ * Corresponding author \\ ** Now at Landesamt für Geoinformation und Landesvermessung Niedersachsen, Germany
}

Keywords: Topographic maps, planetary cartography, map series, atlas

\section{Abstract:}

The High Resolution Stereo Camera (HRSC) of ESA's Mars Express mission [1, 2] is designed to map and investigate the topography of Mars and its satellites. As a push broom scanning instrument with nine CCD line detectors mounted in parallel, its unique feature is the ability to obtain along-track stereo images and four colors during a single orbital pass (Fig. 1). Thus, panchromatic stereo and color images from single orbits of the HRSC have been used to produce digital terrain models (DTMs) and orthoimages of the Martian surface since 2004. The HRSC is still running nominally and delivering new image strips to fill remaining gaps that lead to a contiguous coverage of the surface at high resolution stereo. The very high degree of nadir image coverage (up to 96\%) of the Martian surface is an outstanding characteristic of the Mars Express mission, which no other mission has been achieved so far. The sub-pixel accuracy of derived 3D points results in DTMs with grid sizes of up to $50 \mathrm{~m}$ and height accuracy on the order of one pixel on the ground and better [3].

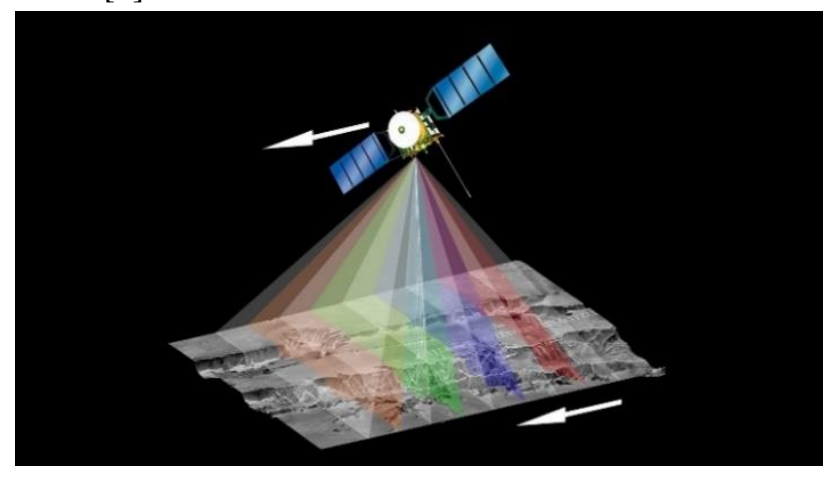

Since 2010 new HRSC multi-orbit data products have been generated, which have been developed into a global mapping program organized into MC-30 half-tiles, since $2014[4,5]$. Based on continuous coverage of an area, regional DTMs and orthomosaics can be produced by combining image data from multiple orbits using specifically adapted techniques for block-adjustment, DTM interpolation and image equalization [6]. The resulting DTMs and color orthomosaics are the baseline for a controlled topographic map series of Mars at a scale of 1:700,000. The extents of the regional products follow the Figure 1. HRSC imaging scheme showing the nine sensors

Batson (Fig. 2) [7], which subdivides Mars into 30 quadrangles. Ronald Greeley and Raymond Batson were pioneers in planetary cartography and set fundamental standards for e.g., map formats and projections for planetary bodies. For the generation of the DTMs and color mosaics, the MC-30 quadrangles are further divided into East (E) and West (W). In parallel to the completion of the first half-tile DTM and color mosaic (MC-11-E) we developed a concept for a topographic map series of Mars [8,9]. To limit data volumes and map sizes, each quadrangle is subdivided into eight tiles (i.e. each half-tile into four tiles, Fig. 3). The map scale of 1:700,000 is a compromise between the high DTM and orthomosaic resolution of $50 \mathrm{~m} / \mathrm{pxl}$ and an acceptable hardcopy format of about $1 \mathrm{~m}$ in width to $2 \mathrm{~m}$ in height. This MC-30 (Mars Chart) global mapping scheme of Greeley and

Figure 2. MC-30 scheme tiling Mars into 30 quadrangles

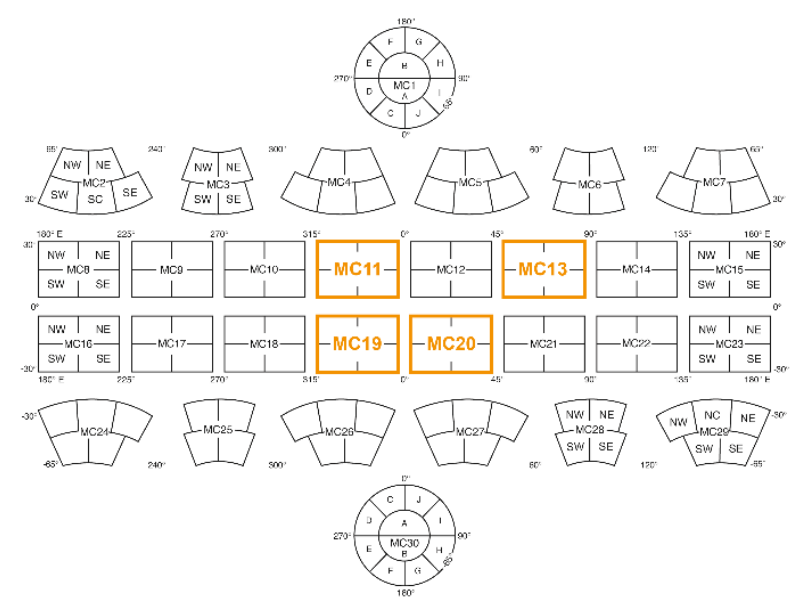




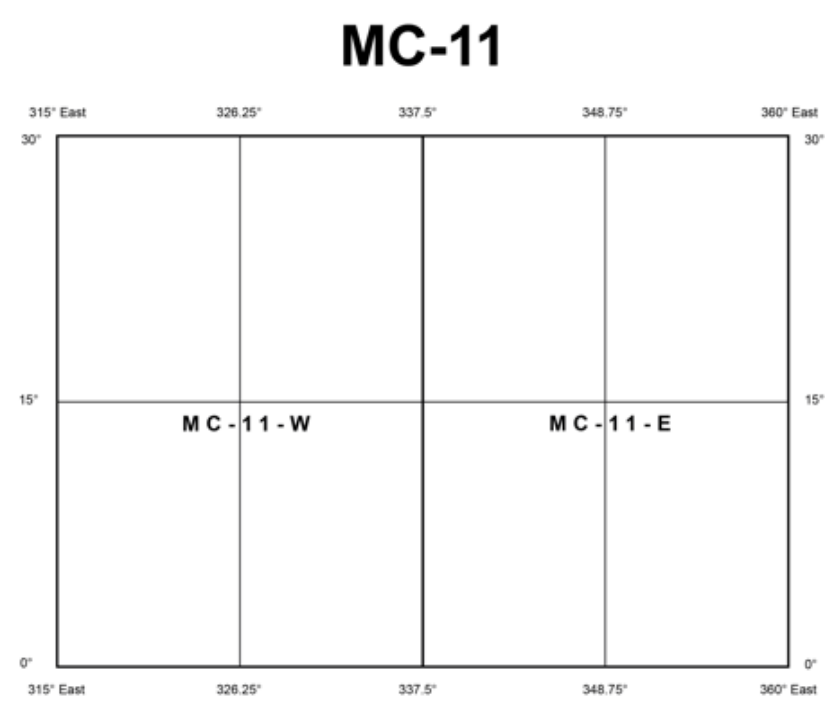

Figure 1. Tiling scheme of the topographic maps results in a printing scale of $14 \mathrm{pxl} / \mathrm{mm}$. MC-11 was selected to be produced first because it contained the four final landing site candidates of ESA's ExoMars mission, including Mawrth Vallis and the finally selected site for the Rosalind Franklin rover (launch in 2022), Oxia Planum. Geographically, the region is located on the northern hemisphere within Acidalia Planitia and Arabia Terra. After MC-11, the Global Topography and Mosaics Task Group (GTMTG) of the HRSC Science Team focussed on MC-13, which is also located on the northern hemisphere and covering parts of Arabia Terra. Further, the quadrangle hosts the landing site of the Perseverance rover from NASA's Mars 2020 mission (arrival in 2021), Jezero crater. Scientists found an ancient river delta near the crater rim that most likely was formed in an ancient crater lake - a highly promising place for the search for life. The next HRSC MC quadrangles will also be equatorial ones (i.e. 19 and 20).

The topographic maps of our series are dominated by the almost format-filling HRSC orthomosaic. Where available, color orthoimages are used, showing Mars-typical reddish-brown colors. The color comes from a combination of the HRSC color channels following a color standard by processing of the mosaic on ground, but is no true color. The cartographic context is given by a geographic coordinate grid with East longitudes from $0^{\circ}$ to $360^{\circ}$ and contour lines that depict the topography of the region. The top layer shows the names of the regions, craters and other landforms that have been named so far. On Mars we mostly have, from large to small, regions (planitia, planum, terra), valleys (vallis), linear features (chasma, fossa, rupes), mountains (mons, tholus), and craters. Also typical for Mars are chaotic terrains (chaos). Besides a text with explanations on the maps and the data we used side maps with a) the footprints of the HRSC image stripes and b) a color-coded and shaded DTM of the shown map tile.

All maps are available for the public at the HRSC team website (http://hrscteam.dlr.de/HMC30/index.html).

\section{Acknowledgements}

We thank the HRSC Experiment team at DLR, Institute of Planetary Research, Berlin, and at Freie Universität Berlin, the HRSC Science Team, as well as the Mars Express Project teams at ESTEC, ESOC, and ESAC for their successful planning and acquisition of data as well as for making processed data available to the HRSC team.

\section{References (optional)}

[1] Neukum, G., Jaumann, R., and The HRSC Co-Investigator Team, HRSC: The High Resolution Stereo Camera of Mars Express. Mars Express: The Scientific Payload, ESA Special Publication, 1240, pp. 17-36, 2004.

[2] Jaumann, R., et al., The high-resolution stereo camera (HRSC) experiment on Mars Express: Instrument aspects and experiment conduct from interplanetary cruise through the nominal mission. Planetary and Space Science 55, pp. 928952, 2007.

[3] Gwinner, K., et al., Topography of Mars from global mapping by HRSC high-resolution digital terrain models and orthoimages: Characteristics and performance, Earth and Planetary Science Letters, 294, pp. 506-519, 2010.

[4] Gwinner, K, et al., Regional HRSC Multi-Orbit Digital Terrain Models for the Mars Science Laboratory Candidate Landing Sites, 41st Lunar and Planetary Science Conference, \#2727, 2010.

[5] Dumke, A., et al., Systematic Processing of High-Resolution Digital Terrain Model Quadrangels on the Basis of MarsExpress HRSC Data, Lunar and Planetary Science Conference, \#1533, 2010.

[6] Gwinner, K. et al., The High Resolution Stereo Camera (HRSC) of Mars Express and its approach to science analysis and mapping for Mars and its satellites, Planetary and Space Science, 126, pp. 93-138, 2016.

[7] Greeley, R. and Batson, G., Planetary Mapping, Cambridge University Press, Cambridge, 1990.

[8] Schulz, K., Topografische Karten der Mars Region MC-11-E, Bachelor Thesis, Beuth Hochschule für Technik Berlin, 2017.

[9] Kersten, E., et al., Topographic mapping of the Mars MC quadrangles using HRSC data. EPSC Abstracts Vol. 12, EPSC2018-352, 2018. 